\title{
Vacuum Sealed Metal Container Closure
}

National Cancer Institute

\section{Source}

National Cancer Institute. Vacuum Sealed Metal Container Closure. NCI Thesaurus. Code C96119.

Metal closures used on packages where the pressure inside the package is less than atmospheric. 